\title{
Paediatric lateral humeral condyle fractures managed by k-wire fixation, should the wires be buried or left exposed: a dilemma
}

\author{
Nitin Choudhary*, Sanjeev Gupta, Neeraj Mahajan, Rahul Mahajan, Amar Saharan
}

Department of Orthopaedics, Govt. Medical College, Jammu, Jammu and Kashmir, India

Received: 05 October 2020

Revised: 03 November 2020

Accepted: 04 November 2020

*Correspondence:

Dr. Nitin Choudhary,

E-mail: nitchy700@gmail.com

Copyright: ( $)$ the author(s), publisher and licensee Medip Academy. This is an open-access article distributed under the terms of the Creative Commons Attribution Non-Commercial License, which permits unrestricted non-commercial use, distribution, and reproduction in any medium, provided the original work is properly cited.

\section{ABSTRACT}

Background: Fractures of the lateral condyle of the humerus in children are common injuries. If displaced or unstable they may require surgical reduction and fixation with Kirschner wires (K-wires). These may be passed through the skin and left exposed or buried subcutaneously.

Methods: A prospective study was carried out in department of orthopaedics at my college from January 2019 to March 2020. A total number of 37 subjects were included in the study.

Results: Patients were followed for a period of 3 months and results were drawn on basis of union, infection, carrying angle at the elbow, and range of motion (ROM) which was assessed by clinico-radiological means.

Conclusions: Buried wire group has shown better union rate than exposed group, wires could be removed under local anaesthesia thereby avoiding any major procedure and also the risk of infection is low making this a better option than the exposed cohort.

Keywords: Lateral condyle humerus fracture, Union, Exposed k-wire, Buried k-wire, Infection

\section{INTRODUCTION}

Fractures of the lateral humeral condyle are common childhood injuries encountered in ortho practice typically caused by a fall onto an extended arm with a varus force. ${ }^{1}$ Un displaced fractures may be treated conservatively with casting, but displaced fractures have a high incidence of non-union, and fractures with more than $2 \mathrm{~mm}$ of displacement require operative fixation. ${ }^{2-5}$ Different methods of fixation have been described; the most widely accepted method is open reduction and fixation with smooth k-wires. ${ }^{2,4,6,10,11}$

K-wires may be buried beneath the skin or inserted through the skin with the wire ends exposed and the literature on the better of the two is still debatable. ${ }^{12-14}$ By their very nature, exposed wires provide a potential portal for infection into the skin and deeper structures. Superficial infections may be treated with a short course of oral antibiotics, but deep infections (septic arthritis/osteomyelitis) may require surgical debridement and a prolonged course of intravenous antibiotics. Reducing the time to removal of exposed k-wires may reduce the risk of infection, but a short duration of fixation may not provide adequate time for secure union to occur. ${ }^{15}$ In contrast, buried wires do not provide an entry point for infection, and wires may be left in situ for extended periods until union can be clearly demonstrated radiographically.

The objective of the current study was to compare the superficial and deep infection rate between exposed and buried $\mathrm{K}$-wires treatment options and to compare the two groups for variables such as postoperative fracture reduction, time to fracture union, elbow range of motion (ROM) and carrying angle, postoperative complications such as infection, non-union, and elbow stiffness. 


\section{METHODS}

This is a prospective study done in department of orthopaedics, government medical college and hospital, Jammu from January 2019 to March 2020.

Inclusion criteria included all children with displaced lateral condylar fractures of distal humerus presenting within a week.

Exclusion criteria excluded open fractures, pathological fractures, delayed presentation ( $>7$ days), un displaced fractures.

Plain radiographs anteroposterior and lateral view of the injured elbow were taken. Fractures were classified as per Milch and Jakob classification system and those that were displaced were subjected to open reduction and k-wire fixation. The k-wires were buried or left exposed as per preoperative categorization and the preference of the operating surgeon.

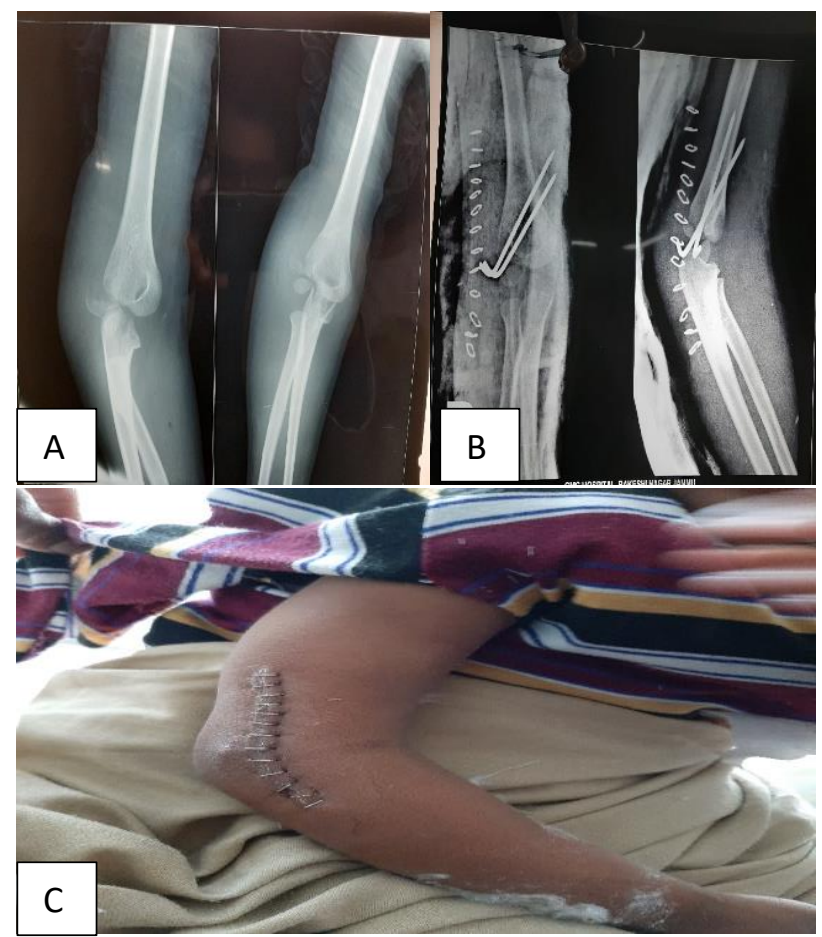

Figure 1: (A, B and C) A child who sustained fracture of lateral condyle of distal humerus managed by buried k-wire fixation.

In our study, in 20 out of 37 subjects the k-wire was buried (group-1) while in other 17 the k-wire was left exposed (group-2). Standard antibiotic protocol of preoperative intravenous injection of $3^{\text {rd }}$-generation cephalosporin and aminoglycoside was given 30 min before surgical incision, followed by $48 \mathrm{~h}$ of same intravenous antibiotic therapy. Postoperative above elbow plaster of Paris slab immobilization was given in all cases and was continued for 6 weeks. X-ray was done on the first postoperative day to assess the fracture reduction, wire placement, and configuration. Fracture reduction was assessed by using Baumann's angle. The patients were discharged on 3rd postoperative day. Subsequently the patients were followed on $7^{\text {th }}$ day, $14^{\text {th }}$ day, 6 weeks, and 3 months. At $7^{\text {th }}$ day follow-up, patients were reviewed for wound inspection. Suture removal and repeat wound inspection were done at 2 weeks. At 6 weeks follow-up, slab radiograph was done to assess fracture reduction, union, and/or radiological signs of osteomyelitis, if any. Superficial infection was managed by short course of oral antibiotics. We tended to remove wire at 6 weeks in both groups. In our study we removed buried wires under local anaesthesia in outpatient setting. At 3 months follow-up, the following parameters were assessed clinicoradiologically; status of fracture reduction, status of union, any evidence of osteomyelitis, carrying angle at the elbow, and elbow ROM.

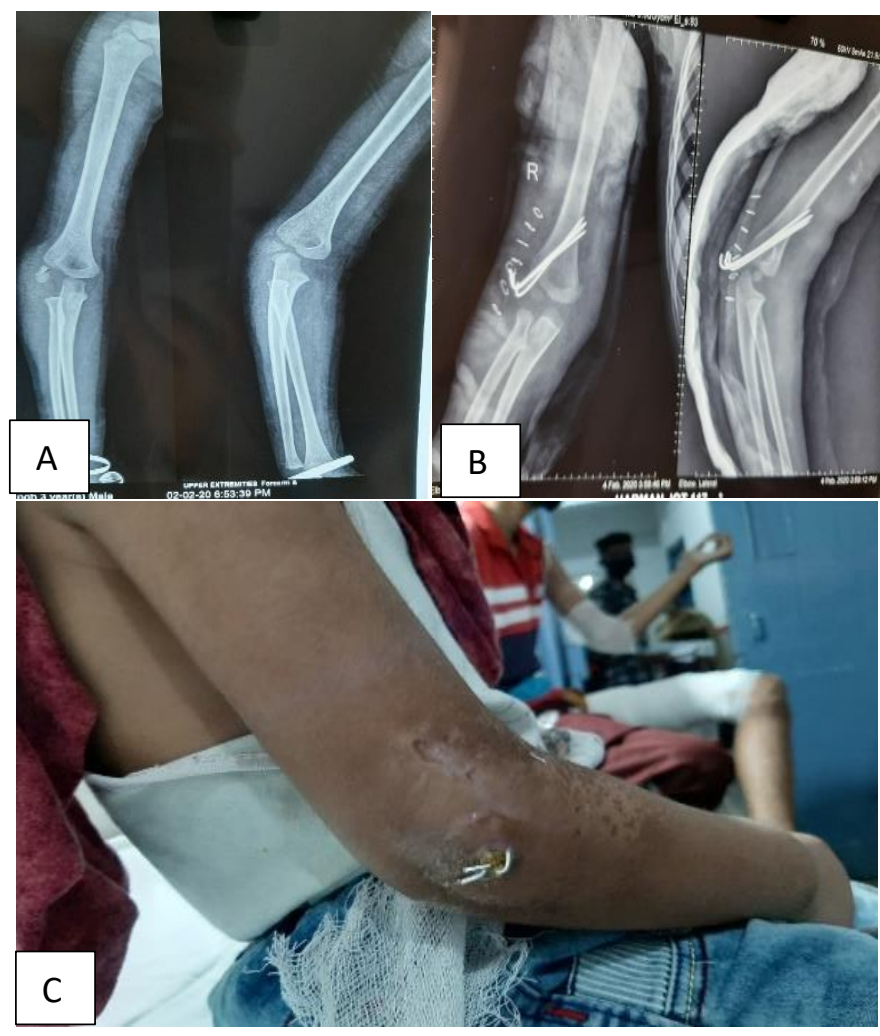

Figure 2: (A, B and C) A child with lateral condyle fracture of distal humerus who has been managed by exposed k-wire fixation.

Data was collected and analysed with help of IBM SPSS software (version 20.0). $\mathrm{P}$ values $<0.05$ are considered to be statistically significant.

The study was approved by the institutional ethics committee.

\section{RESULTS}

Out of 37 patients 25 were males and 12 were females. Mean age was $6.78 \pm 2.47$ years and the most common 
mechanism of injury was falling while playing. Both groups were compared in terms of outcome on basis of Baumann's angle, time to union, range of motion, carrying angle, infection.

Table 1: Comparison between outcome of two groups on basis of various characteristics.

\begin{tabular}{|c|c|c|c|}
\hline Characteristic & Group-1 & Group-2 & $\mathbf{P}$ \\
\hline Infection & 0 & 3 & 0.06 \\
\hline $\begin{array}{l}\text { Baumann's } \\
\text { angle at } 3^{\text {rd }} \\
\text { month }\left(^{\circ}\right)\end{array}$ & $72 \pm 9.67$ & $69 \pm 8.25$ & 0.78 \\
\hline Time to union & $5.16 \pm 0.51$ & $5.5 \pm 0.34$ & 0.037 \\
\hline $\begin{array}{l}\text { Elbow flexion } \\
\left({ }^{\circ}\right)\end{array}$ & $134.76 \pm 3.90$ & $\begin{array}{l}134.04 \pm 5.3 \\
7\end{array}$ & 0.590 \\
\hline $\begin{array}{l}\text { Extensor lag } \\
\text { elbow }\left({ }^{\circ}\right)\end{array}$ & $3.44 \pm 7.39$ & $3.89 \pm 6.54$ & 0.67 \\
\hline $\begin{array}{l}\text { Lateral } \\
\text { condylar } \\
\text { overgrowth }\end{array}$ & 2 & 3 & 0.08 \\
\hline $\begin{array}{l}\text { Carrying angle } \\
\left({ }^{\circ}\right)\end{array}$ & $6.98 \pm 5.43$ & $4.68 \pm 2.48$ & 0.12 \\
\hline
\end{tabular}

The mean Baumann's angle in group 1 patients at 3 months follow up was $72 \pm 9.67$ and in group 2 patients was $69 \pm 8.25$. The $p$ value was insignificant. The elbow flexion in group 1 and 2 patients was 134.76 \pm 3.90 and $134.04 \pm 5.37$ respectively. 2 and 3 cases of lateral condylar overgrowth were observed in $1^{\text {st }}$ and $2^{\text {nd }}$ group respectively. The extensor lag and carrying angle was also measured at 3 months for comparison however the values were found to be insignificant, hence no difference on the above variable/characteristic was seen on account of leaving wires exposed or burying them.

As for time to union was concerned the average time to union was $5.16 \pm 0.51$ weeks in group 1 patients and $5.5 \pm 0.34$ weeks in group 2 patients. The difference was found to be statically significant $(\mathrm{p}=0.037)$. Two patients in group 2 had not shown in radiographs had not shown union at 6 weeks follow up radiograph and therefore wires were removed at a later time than usual in these two cases. The reason for this may be sighted as regarding the adequacy of fixation of exposed wires. Buried wires are bent close to the bone, and the bend in the wire prevents the fracture fragment sliding along the wire and displacing while in exposed wires bent is at a distance from the bone which may cause sliding of bone fragment, also displacement of wires during ASD and suture removal may alter the compression achieved at the site of fracture specially if being done by some untrained person at some periphery (a common scenario for most patients from far off places who wish to get dressing and suture removal done in their own local area). However, both these patients had normal clinico-radiological characteristics at 3 months follow-up.

We encountered three cases of infection in exposed group and none in buried group. There was no case of any skin related complication in buried group. All three infections in exposed group were superficial which were cleared by oral antibiotics and adequate pin site hygiene.

\section{DISCUSSION}

There is a paucity in literature comparing outcome in exposed versus buried $\mathrm{K}$-wires fixation in lateral condyle fractures. In our study males $(68 \%)$ had higher percentage than females $(32 \%)$ with fall while playing being the most common mechanism.

Infection rate was more in superficial group than in deep group and this corresponds to the study done by Launay et al. ${ }^{12}$ However, Ormsby and Siow et al noted lower infection rates in exposed cohort, this however may be attributed to technique and antibiotic prophylaxis and also non-randomized nature of the sudy. ${ }^{16,17}$

The wires were removed uniformly at 6 weeks except for two cases where they were removed later. Thomas et al ${ }^{18}$ advocated 3 weeks of $\mathrm{k}$-wire stabilization and began elbow mobilization after the elapse of this period. They reported one case of delayed union in a patient whose k-wires were removed at 19 days. So, in our study, early removal of kwires before 6 weeks was not done in any patient.

Radiologically, lateral condyle overgrowth was seen in 11 cases in Group-1 and 12 cases in Group-2 with no statistically significant difference. It did not produce any substantial functional problems with elbow motion. This complication was reported by Launay and Chan et al and they showed high rate of lateral condyle overgrowth in exposed as compared to buried. ${ }^{12,17}$ Launay et al believed that prolonged postoperative immobilization seems to contribute to the formation of lateral condyle overgrowth and occurred significantly with postoperative immobilization for 6 weeks compared immobilization for $<6$ weeks (78.0 vs. $42.9 \%$, respectively). ${ }^{12}$ However, this reason seems inappropriate for our study as immobilization period was same for both groups.

Time to union was different in both groups and was found to be statically significant. Two patients of exposed group had wires removed at around 7 weeks, one was Jakob type 2 and other was type 3. Many studies have documented earlier union with buried group, however, recently studies have shown exposed wires to be as good as buried as far as union is concerned, also keeping in mind the additional procedure that is required to remove the buried wires. But we in our study in all our subjects were able to remove wires under local anaesthesia. Also, if proper limb elevation is maintained both pre as well as post op the chances of infection or skin problems with buried group is almost null.

In both groups the outcome in terms of Baumann's angle and carrying angle at 3 months follow up to be statically insignificant. Chan and Siow ${ }^{17}$ observed similar results in 72 cases. Launay et al reported loss of flexion ranges from 
$0^{\circ}$ to $25^{\circ}$, loss of extension ranges from $0^{\circ}$ to $30^{\circ} \cdot{ }^{12}$ Elbow ROM was fairly preserved in all cases in our study.

\section{CONCLUSION}

Both techniques have their merits and de-merits. However, buried wire group have shown better union rate than exposed group, wires could be removed under local anaesthesia thereby avoiding any major procedure and also the risk of infection is low making this a better option than the exposed cohort.

Funding: No funding sources

Conflict of interest: None declared

Ethical approval: The study was approved by the institutional ethics committee

\section{REFERENCES}

1. Jakob R, Fowles JV, Rang M, Kassab MT. Observations concerning fractures of the lateral humeral condyle in children. J Bone Joint Surg. 1975;57(4):430-6.

2. Flynn JC, Richards JF. Non-union of minimally displaced fractures of the lateral condyle of the humerus in children. J Bone Joint Surg Am. 1971;53(6):1096-1101.

3. Badelon O, Bensahel H, Mazda K, Vie P. Lateral humeral condylar fractures in children: a report of 47 cases. J Pediatr Orthop. 1988;8(1):31-4.

4. Bast SC, Hoffer MM, Aval S. Nonoperative treatment for minimally and nondisplaced lateral humeral condyle fractures in children. J Pediatr Orthop. 1998;18(4):448-50.

5. Foster DE, Sullivan JA, Gross RH. Lateral humeral condylar fractures in children. J Pediatr Orthop. 1985;5(1):16-22.

6. Conner AN, Smith MG. Displaced fractures of the lateral humeral condyle in children. J Bone Joint Surg Br. 1970;52(3):460-64.

7. Mintzer CM, Waters PM, Brown DJ, Kasser JR. Percutaneous pinning in the treatment of displaced lateral condyle fractures. J Pediatr Orthop. 1994;14(4):462-5.

8. Song KS, Kang CH, Min BW, Bae KC, Cho CH, Lee $\mathrm{JH}$ et al. Closed reduction and internal fixation of displaced unstable lateral condylar fractures of the humerus in children. J Bone Joint Surg Am. 2008;90(12):2673-81.

9. Hausman MR, Qureshi S, Goldstein R, Langford J, Klug RA, Radomisli TE et al. Arthroscopically- assisted treatment of pediatric lateral humeral condyle fractures. J Pediatr Orthop. 2007;27(7):739-42.

10. Sharma JC, Arora A, Mathur NC, Gupta SP, Biyani A, Mathur R et al. Lateral condylar fractures of the humerus in children: fixation with partially threaded 4.0-mm AO cancellous screws. J Trauma. 1995;39(6):1129-33.

11. Flynn JC, Richards JF, Saltzman RI. Prevention and treatment of non-union of slightly displaced fractures of the lateral humeral condyle in children. An endresult study. J Bone Joint Surg Am. 1975;57(8):108792.

12. Launay F, Leet AI, Jacopin S, Jouve JL, Bollini G, Sponseller PD et al. Lateral humeral condyle fractures in children: a comparison of two approaches to treatment. J Pediatr Orthop. 2004;24(4):385-91.

13. Beaty JH, Kasser J. Lateral condyle fractures in children. In: John M Flynn, David L Skaggs, Peter M Waters(eds). Rockwood and Wilkins' fractures in children, $8^{\text {th }}$ edn, Wolters Kluwer, Philadelphia 2015;781-834.

14. Morrissy RT, Weinstein SL. Open reduction and internal fixation of displaced lateral condyle fracture of the humerus. In: Weinstein SL, Morrissy RT (eds) Atlas of paediatric orthopaedic surgery, $4^{\text {th }}$ edn. Lippincott Williams and Wilkins, Philadelphia,2006;73-9.

15. Cardona JI, Riddle E, Kumar SJ. Displaced fractures of the lateral humeral condyle: criteria for implant removal. J Pediatr Orthop. 2002;22(2):194-7.

16. Ormsby NM, Walton RD, Robinson S, BrookesFazakerly S, Chang FY, McGonagle L et al. Buried versus unburied Kirschner wires in the management of paediatric lateral condyle elbow fractures: A comparative study from a tertiary centre. J Pediatr Orthop B. 2016;25:69-73.

17. Chan LW, Siow HM. Exposed versus buried wires for fixation of lateral humeral condyle fractures in children: A comparison of safety and efficacy. J Child Orthop. 2011;5:329-33.

18. Thomas DP, Howard AW, Cole WG, Hedden DM. Three weeks of kirschner wire fixation for displaced lateral condylar fractures of the humerus in children. J Pediatr Orthop. 2001;21:565-9.

Cite this article as: Choudhary N, Gupta S, Mahajan N, Mahajan R, Saharan A. Paediatric lateral humeral condyle fractures managed by kwire fixation, should the wires be buried or left exposed: a dilemma. Int J Res Orthop 2021;7:78-81. 